\title{
Linear optics control of sideband instability for improved free-electron laser spectral brightness
}

\author{
G. Perosa, ${ }^{1}$ E. M. Allaria, ${ }^{2}$ L. Badano, ${ }^{2}$ N. Bruchon $\odot,{ }^{3}$ P. Cinquegrana, ${ }^{2}$ M. B. Danailov, ${ }^{2}$ \\ A. Demidovich $\odot{ }^{2}$ G. De Ninno, ${ }^{2,4}$ L. Giannessi ${ }^{2,5}$ N. Mirian $\odot,{ }^{2}$ G. M. Penco, ${ }^{2}$ P. Rebernik $\odot,{ }^{2,4}$ \\ E. Roussel, ${ }^{6}$ P. Sigalotti, ${ }^{2}$ S. Spampinati, ${ }^{2}$ M. Veronese $\odot,{ }^{2}$ M. Trovò, ${ }^{2}$ and S. Di Mitri® ${ }^{1,2, *}$ \\ ${ }^{1}$ Department of Physics, Università degli Studi di Trieste, 34127 Trieste, Italy \\ ${ }^{2}$ Elettra-Sincrotrone Trieste S.C.p.A., 34149 Basovizza, Trieste, Italy \\ ${ }^{3}$ Department of Engineering and Architecture, Università degli Studi di Trieste, 34127 Trieste, Italy \\ ${ }^{4}$ Laboratory of Quantum Optics, University of Nova Gorica, Nova Gorica, Slovenia \\ ${ }^{5}$ Istituto Nazionale di Fisica Nucleare, Laboratori Nazionali di Frascati, 00044 Frascati, Italy \\ ${ }^{6}$ Université de Lille, CNRS, UMR 8523, Physique des Lasers Atomes et Molécules, Lille, France
}

(Received 17 July 2020; accepted 14 October 2020; published 17 November 2020)

\begin{abstract}
Extension of stable longitudinal coherence from vacuum ultraviolet to $\mathrm{x}$ rays is highly sought after in the free-electron laser (FEL) community, but it is often prevented by bandwidth broadening originated in the electron beam microbunching instability. We demonstrate that a proper tuning of the linear optics before the beam enters the undulator mitigates the microbunching-induced sideband instability. The experiment was conducted at the Fermi FEL operated in echo-enabled harmonic generation mode, where the spectral brightness at $7 \mathrm{~nm}$ wavelength was doubled. The FEL performance is compared to nonoptimized optics solutions and characterized in terms of peak intensity and spectral bandwidth shot-to-shot stability. The technique has straightforward implementation, because it uses quadrupole magnets routinely adopted for beam transport, and it applies to any FEL architecture, so paving the way to the production of high-intensity Fourier-transform limited x-ray pulses in existing and planned FEL facilities.
\end{abstract}

DOI: 10.1103/PhysRevAccelBeams.23.110703

\section{INTRODUCTION}

UV and x-ray free-electron lasers (FELs) have become invaluable tools for the exploration of matter [1-4]. Using $\mathrm{x}$ rays to perform a broad variety of four-wave mixing (FWM) spectroscopies is of particular importance [5-8], as the multiwave nature of FWM enables probing processes and correlations involving different states of the system. The use of highly brilliant photon pulses allows one to monitor the dynamics of such a wealth of processes in real time and on timescales ranging, e.g., from slow (microsecond) structural relaxations to ultrafast (subpicosecond) chemical reactions $[9,10]$. On top of exquisite synchronization and high intensities, temporal coherence of the FEL pulses, which commonly translates into narrow spectral bandwidth, is an essential requirement to FWM experiments [11].

Self-amplified spontaneous emission FELs [12,13] present a multispiky spectrum with little longitudinal

"simone.dimitri@elettra.eu

Published by the American Physical Society under the terms of the Creative Commons Attribution 4.0 International license. Further distribution of this work must maintain attribution to the author(s) and the published article's title, journal citation, and DOI. coherence. This is improved in x-ray self-seeding FELs, but at the expense of large shot-to-shot intensity fluctuations [14-16]. Stable longitudinal coherence, i.e., a reproducible single spectral line, is to date produced only at externally seeded vacuum ultraviolet (VUV) FELs operating in high gain harmonic generation [17-20] and echoenabled harmonic generation (EEHG) $[21,22]$ scheme.

The possibility of extending stable longitudinal coherence to $\mathrm{x}$-ray photon energies $(>300 \mathrm{eV})$, although highly sought after in the light source user community [23], is often prevented by broadband electron beam energy and density modulations resulting from microbunching instability [24,25]. The instability captures and amplifies modulations up to a few hundreds of micrometer wavelength in the initial beam distribution [26]. These are blueshifted by bunch length compression, typically by a factor of 10-100, to the scale of the FEL cooperation length [27], e.g., in the range $0.1-1 \mu \mathrm{m}$ for hard $\mathrm{x}$ rays, and up to a few micrometers in VUV and soft $x$ rays. The mix of frequencies induced by microbunching instability and by the FEL coherent emission generates shot-to-shot fluctuations of the multiline FEL spectrum [28-30]. In a seeded FEL, this can be interpreted as the stochastic appearance of sidebands at frequencies [31]

$$
k_{\mathrm{FEL}}=h k_{\mathrm{seed}} \pm m k_{\mathrm{MBI}} \text {, }
$$


where, for example, $h \sim 36$ is the FEL harmonic jump from the seed laser wave number $k_{\text {seed }} \sim 2 \pi / 260 \mathrm{~nm}^{-1}, m \sim 10$ is the bunch length compression factor, and $k_{\mathrm{MBI}} \sim$ $2 \pi / 50 \mu \mathrm{m}^{-1}$ is the instability wave number evaluated before compression. The spectral broadening averaged over many shots is sometimes referred to as a "pedestal."

Among several strategies proposed to mitigate the instability [32-35], several facilities have chosen energy Landau damping [36-38]: The electron beam at $\sim 100 \mathrm{MeV}$ energy interacts with an infrared laser in a short undulator, the socalled laser heater [39], which adds up to a few tens of $\mathrm{keV}$ uncorrelated energy spread to the beam before the instability builds up. Transverse shaping of the laser beam promises further improvement of the FEL performance $[40,41]$. Nevertheless, the laser heater power is deliberately limited to avoid depleting the FEL gain; this leads in some configurations to deal with residual microbunching. In cases where a very narrow FEL bandwidth is desired, the laser heater power is strongly increased, at the expense of the output intensity. In short, the optimum laser heater intensity often consists in a balance of FEL spectral purity and high pulse energy. Demanding experiments can require both. Strategies for the mitigation of the microbunching instability which do not rely on an increase of the beam energy spread would allow high-intensity FEL pulses and a stable, very narrow bandwidth. The investigation of a solution of this kind is the subject of this article. We show that linear optics control can be an additional knob, complementary to the laser heater, for mitigation of the microbunching instability. In particular, we report for the first time mitigation of the FEL sideband instability by tuning the momentum compaction (or $R_{56}$ ) of a transfer line upstream of the undulator (or "spreader") to an isochronous condition (henceforth identified by the condition $R_{56}=0$ ). The study was conducted at the Fermi FEL operated in EEHG mode and lasing at the fundamental wavelength of $7.3 \mathrm{~nm}[22]$.

\section{EXPERIMENT}

The solution $R_{56}=0$ was built following the prescriptions in Ref. [42] for minimization of the coherent synchrotron radiation (CSR)-induced microbunching; i.e., the line was made globally and locally isochronous $\left(R_{56}\right.$ is also zeroed at intermediate locations along the line) and with small absolute values of the local $R_{56}$. In short, this design relies on the minimization of the term:

$$
\xi=\left|\max \left\{R_{56}^{s^{\prime} \rightarrow s}\right\} \frac{k^{1 / 3}}{\rho^{2 / 3}} \Delta L\right|,
$$

which drives the CSR instability [43,44] in a multibend line. $R_{56}^{s^{\prime} \rightarrow s}$ is the value of $R_{56}$ evaluated at consecutive dipole locations $s^{\prime}$ and $s$ along the beam line, $\rho$ the dipole bending radius, and $\Delta L=s^{\prime}-s$.
TABLE I. List of Fermi parameters (measured).

\begin{tabular}{|c|c|c|}
\hline Linac & Value & Units \\
\hline Bunch charge & 0.7 & $\mathrm{nC}$ \\
\hline Initial peak current & 70 & A \\
\hline Initial normalized emittance $(\mathrm{x}, \mathrm{y})$ & $0.9,0.8$ & $\mu \mathrm{m} \mathrm{rad}$ \\
\hline Beam energy at $\mathrm{BC} 1$ & 0.29 & $\mathrm{GeV}$ \\
\hline$R_{56}$ of $\mathrm{BC} 1$ & -41.9 & $\mathrm{~mm}$ \\
\hline Compression factor & 10 & \\
\hline Final beam energy & 1.31 & $\mathrm{GeV}$ \\
\hline Final energy spread, rms & $<0.1$ & $\%$ \\
\hline Final linear energy chirp & $<6$ & $\mathrm{MeV} / \mathrm{ps}$ \\
\hline Final normalized emittance $(\mathrm{x}, \mathrm{y})$ & $2.2,1.8( \pm 0.2)$ & $\mu m \mathrm{rad}$ \\
\hline \multicolumn{3}{|l|}{ Spreader } \\
\hline Dipole bending angle & 52 & $\operatorname{mrad}$ \\
\hline Dipole length & 0.4 & $\mathrm{~m}$ \\
\hline Total $R_{56}$ & $-2,0,2$ & $\mathrm{~mm}$ \\
\hline Maximum local $\left|R_{56}\right|$ & $2,0.2,2$ & $\mathrm{~mm}$ \\
\hline Final normalized emittance (x) & $2.2( \pm 0.2)$ & $\mu m \mathrm{rad}$ \\
\hline Final normalized emittance (y) & $1.9( \pm 0.2)$ & $\mu m \mathrm{rad}$ \\
\hline \multicolumn{3}{|l|}{ Seed lasers } \\
\hline Central wavelength & 264 & $\mathrm{~nm}$ \\
\hline Pulse energy & $<50$ & $\mu \mathrm{J}$ \\
\hline Pulse duration, FWHM & $\sim 150 / \sim 130$ & fs \\
\hline Peak power & $43 / 90$ & MW \\
\hline \multicolumn{3}{|l|}{ EEHG FEL } \\
\hline Radiator, period length & 3.5 & $\mathrm{~cm}$ \\
\hline Radiator, number of periods & $6 \times 68$ & \\
\hline First dispersive section, $\left|R_{56}\right|$ & 2.38 & $\mathrm{~mm}$ \\
\hline Second dispersive section, $\left|R_{56}\right|$ & 0.06 & $\mathrm{~mm}$ \\
\hline Central wavelength & 7.3 & $\mathrm{~nm}$ \\
\hline FEL pulse energy, maximum value & $\sim 25$ & $\mu \mathrm{J}$ \\
\hline FEL pulse duration, FWHM & $\sim 60$ & fs \\
\hline FEL gain length & 1.9 & $\mathrm{~m}$ \\
\hline
\end{tabular}

The list of parameters adopted during the experiment is given in Table I. Each branch of the Fermi spreader line is made of two double bend achromatic cells separated by quadrupole magnets. The EEHG spectrum and intensity was recorded for three values of the spreader momentum compaction, i.e., $R_{56} \cong-2,0,+2 \mathrm{~mm}$ (four-dipole chicanes have negative $R_{56}$ with this convention). The linear optics functions are shown in Fig. 1, and the $R_{56}$ along the line is in Fig. 2. For each setting of the quadrupole magnets devoted to produce a specific $R_{56}$ value, the FEL intensity was reoptimized as a function of the electron beam optical Twiss parameters at the entrance of the undulator, seed laser parameters, and strength of the dispersive sections of the EEHG scheme.

By virtue of the small correlated energy spread at the entrance of the spreader $(<0.1 \%$, rms value $)$, the bunch length differences between datasets due to first- and higherorder transport matrix terms were negligible $(<2 \%)$. Finally, the spreader optics was tuned to also preserve the beam bend-plane emittance from CSR emitted in the dipoles [45]. The final emittance values reported in Table I 

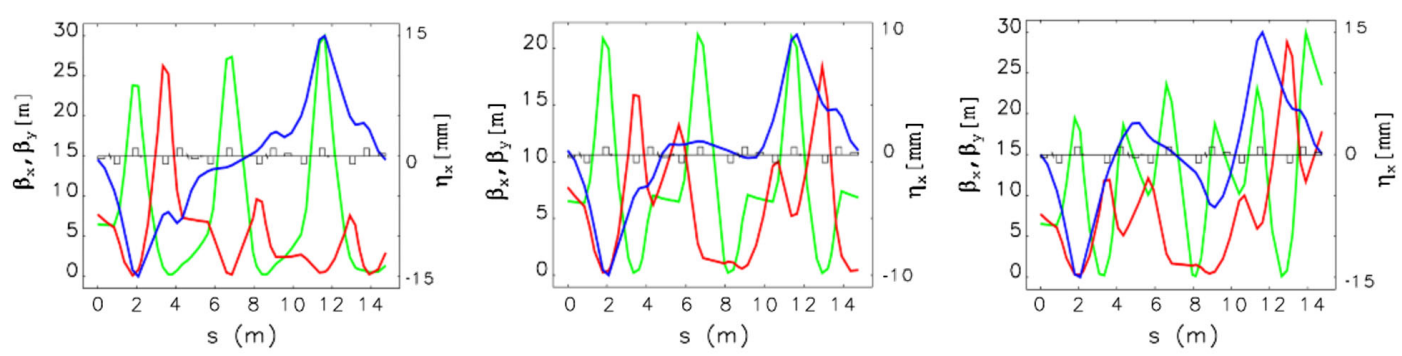

FIG. 1. Betatron functions (x, green line; y, red line) and horizontal energy dispersion function (blue line) along the Fermi spreader, for $R_{56}=+2 \mathrm{~mm}$ (left), 0 (middle), and $-2 \mathrm{~mm}$ (right). In gray, big rectangles are quadrupole magnets, and small rectangles are dipole magnets.

are the largest values among all the measurements, which were repeated at each change of $R_{56}$.

The EEHG output was recorded on a single-shot basis at the on-line spectrometer. In the following, the FEL data refer to the average of 41 consecutive FEL shots recorded at $50 \mathrm{~Hz}$. Each dataset was measured as a function of the laser heater pulse energy, as shown in Fig. 3(a). The contribution of the pedestal to the spectrum was evaluated by fitting the central FEL line with a Gaussian. The area of the Gaussian was subtracted from the total integrated spectrum signal. Doing so, we calculated the ratio of the pedestal signal $\left(A_{\mathrm{SB}}\right)$ and the total FEL signal $\left(A_{\mathrm{tot}}\right)$, as shown in Fig. 3(b). Finally, Fig. 3(c) shows the ratio of the FEL total signal and the spectral bandwidth. The latter quantity is the width of the spectrum image, corresponding to the interval containing $76 \%$ of the central profile area, in analogy to a Gaussian FWHM.

Since the FEL signal is proportional to the pulse energy, and since the spatial, angular, and temporal width of the electron beam in the undulator does not change as the laser heater is varied, the quantity in Fig. 3(c) is proportional to the FEL spectral brightness (brilliance). In all plots, the error bars are dominated by fluctuations driven by electron beam shot-to-shot jitter. A closer look at the FEL spectrum at low and moderate laser heater pulse energy is given in Fig. 4.

Figure 3 highlights the improved spectral purity when passing from positive to null to negative $R_{56}$ of the spreader

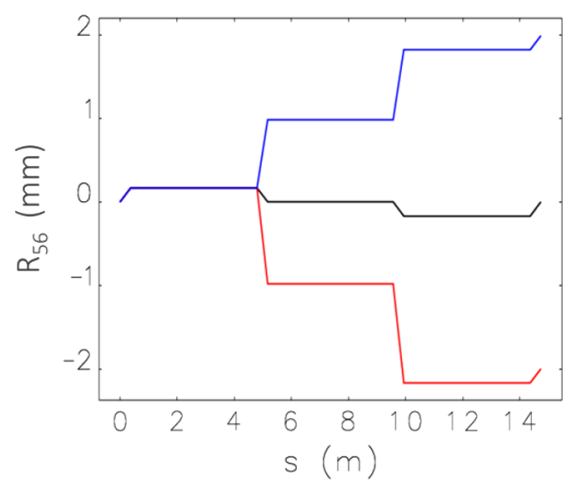

FIG. 2. $R_{56}$ along the spreader for the three optics in Fig. 1. line. In the latter case, however, there is no net increase of the brilliance compared to the isochronous optics, and the FEL intensity is reduced at the front of a higher spectral purity. The evident stronger dependence of the FEL
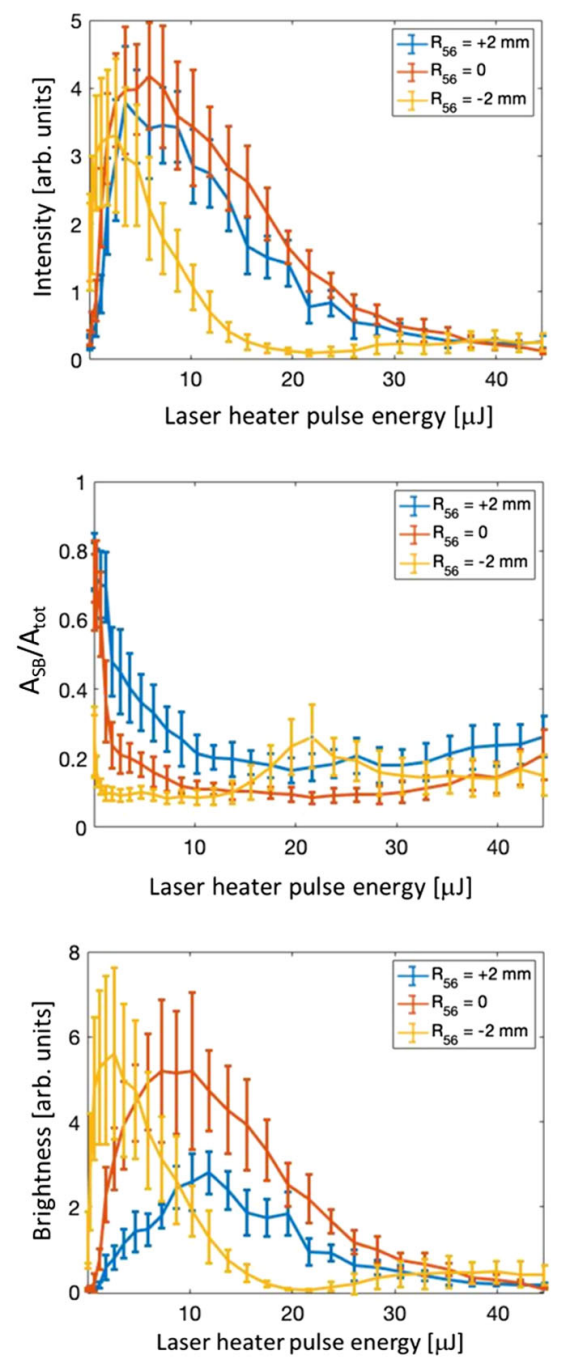

FIG. 3. EEHG signal recorded at $7.3 \mathrm{~nm}$, for the three spreader optics in Fig. 1, as a function of the laser heater pulse energy. From top to bottom: Total signal; ratio of the pedestal signal over the total FEL signal; total signal over FWHM bandwidth. All quantities are in arbitrary units. 


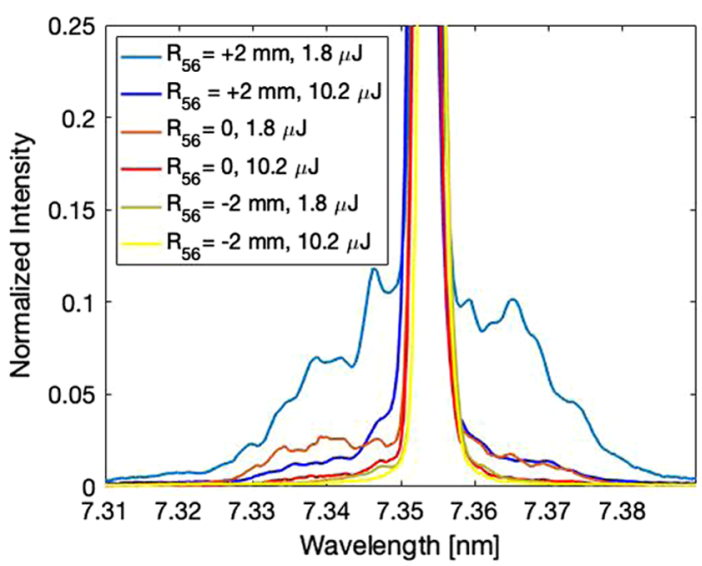

FIG. 4. EEHG spectrum (average over 31 shots) for the three spreader optics in Fig. 1, at two laser heater pulse energies.

intensity from the laser heater ( $\mathrm{LH}$ ) for the negative $R_{56}$ compared to the other two cases might suggest an abrupt change of the experimental conditions, e.g., of the LH operational parameters. This has not been revealed by an analysis of the machine setting. Nevertheless, and in order to disentangle the results from the actual LH setting, an additional analysis is conducted by comparing the amount of relative FEL pulse energy distributed into the sidebands at the point of maximum FEL brilliance, i.e., for the LH setting that optimizes the FEL performance in each of the three experimental sessions. The same quantity is also calculated for the LH turned off. The results are shown in Fig. 5.

The trend of the experimental data with $R_{56}$ is corroborated by a semianalytical prediction based on the model for the microbunching instability introduced in Ref. [46]. This allows us to follow the instability from the Fermi injector to the spreader end, by including a two-dimensional modeling of the laser heater effect [36], and an intrabeam scattering theory revisited for single-pass systems [47]. The model

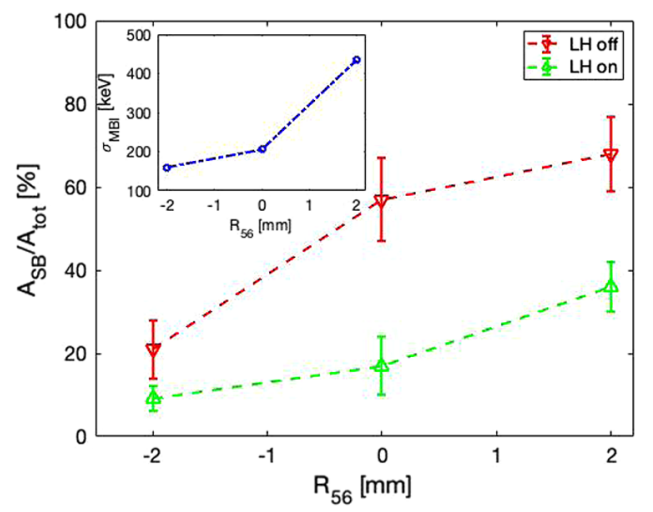

FIG. 5. Measured average spectral area of sidebands with the laser heater turned off (red triangles) and on (green triangles), the latter being the value which maximizes the FEL brilliance. In the inset, predicted $\sigma_{\mathrm{MBI}}$ calculated for the laser heater off. takes into account the coupled dynamics of density and energy modulations, i.e., the successive translation of bunching into energy modulation through longitudinal space charge and CSR impedance and of energy modulations into bunching via $R_{56}$ terms [48].

Since the instability is broadband, but only modulation periods in the range $\sim 1-10 \mu \mathrm{m}$ are expected to have a substantial impact on the FEL bandwidth [see Eq. (1)], we collapsed the information of the microbunching instability "strength" into the spectral integral of the energy modulation curve in the aforementioned wavelength range, weighted by the instability gain $[47,49]$. We refer to this quantity as $\sigma_{\mathrm{MBI}}$. Although the calculated $\sigma_{\mathrm{MBI}}$ cannot be directly related to the measured FEL spectral purity, the weaker the instability gain is, the lower the $\sigma_{\mathrm{MBI}}$ is, and the smaller the amount of FEL energy is expected to be dispersed into sidebands.

\section{DISCUSSION}

The microbunching instability model suggests an interpretation of the experimental results for the negative $R_{56}$ based on phase mixing [34]. When the beam reaches the spreader with a density modulation generated by the instability in the accelerator - this is the case of laser heater turned off-the longitudinal slippage of particles, generated by the nonzero $R_{56}$ times the local energy chirp at the modulation scale, smoothens the beam longitudinal phase space. This translates into a redshift of the peak gain toward wavelengths that do not interference anymore with the FEL natural bandwidth. This picture is consistent with theoretical and numerical results reported also in Refs. [50,51].

Contrary to energy Landau damping, the effect of phase mixing depends on the sign of $R_{56}$, which explains the asymmetry of the measured areas for positive and negative $R_{56}$ values in Fig. 5. In general, the phase mixing has an optimal effect, i.e., $R_{56}$ is at the optimum nonzero value, when the most harmful modulations incoming from the accelerator are smoothened out. Increasing further $R_{56}$ would not provide any additional benefit, while enhancing the CSR-induced MBI due to the nonisochronous optics. Therefore, an optimum value of $R_{56}$ is expected depending upon the actual machine configuration and the FEL wavelength range under consideration.

We finally observe that, when the laser heater is turned on, phase mixing is less effective, and the sideband area in Fig. 5 decreases only from $\sim 40 \%$ to $\sim 10 \%$ across the full $R_{56}$ scan, compared to a reduction from $\sim 70 \%$ to $\sim 20 \%$ when the heater is off.

\section{CONCLUSIONS}

We have reported a characterization of the Fermi EEHG FEL emission as a function of the linear momentum compaction of the spreader line. The isochronous optics provides both a higher FEL signal and a narrower 
bandwidth, compared to a positive value of $R_{56}$, by minimizing the CSR-induced microbunching instability gain. An even higher spectral purity is observed at negative $R_{56}$, but this effect becomes more evident at lower laser heater values and with no net improvement of the FEL pulse energy. Our findings are interpreted in the light of a revisited semianalytical model of the instability through the whole beam line, which confirms that a complementary action of laser heater and linear optics can substantially increase the FEL spectral brightness.

The technique has straightforward implementation, because it uses quadrupole magnets routinely adopted for beam transport. Since it applies to any FEL architecture, and owing to the notorious development of microbunching instability in high-brightness electron linacs, the technique finds application in nonseeded as well as externally and self-seeded FELs. In particular, it supports the capability of externally seeded FELs to produce stable, full longitudinal coherence at x-ray photon energies.

\section{ACKNOWLEDGMENTS}

The authors acknowledge the continuous support of the Fermi team and the Elettra Sincrotrone Trieste operators during the experiment.

[1] C. Pellegrini, The physics of x-ray free-electron lasers, Rev. Mod. Phys. 88, 015006 (2016).

[2] E. A. Seddon et al., Short-wavelength free-electron laser sources and science: a review, Rep. Prog. Phys. 80, 115901 (2017).

[3] R. Schonlein, T. Elsaesser, K. Holldack, Z. Huang, H. Kapteyn, M. Murnane, and M. Woerner, Recent advances in ultrafast x-ray sources, Phil. Trans. R. Soc. A 377, 20180384 (2019).

[4] S. Di Mitri and M. Cornacchia, Electron beam brightness in linac drivers for free-electron-lasers, Phys. Rep. 539, 1 (2014).

[5] X. Li, P. L. Voss, J. E. Sharping, and P. Kumar, OpticalFiber Source of Polarization-Entangled Photons in the $1550 \mathrm{~nm}$ Telecom Band, Phys. Rev. Lett. 94, 053601 (2005).

[6] M. A. Foster, A. C. Turner, J. E. Sharping, B. S. Schmidt, M. Lipson, and A. L. Gaeta, Broad-band optical parametric gain on a silicon photonic chip, Nature (London) 441, 960 (2006).

[7] D. Avisar and D. J. Tannor, Complete Reconstruction of the Wave Function of a Reacting Molecule by Four-Wave Mixing Spectroscopy, Phys. Rev. Lett. 106, 170405 (2011).

[8] S. Mukamel, D. Healion, Y. Zhang, and J. D. Biggs, Multidimensional attosecond resonant X-Ray spectroscopy of molecules: Lessons from the optical regime, Annu. Rev. Phys. Chem. 64, 101 (2013).

[9] N. Bloembergen, Nonlinear optics and spectroscopy, Rev. Mod. Phys. 54, 685 (1982); L. Dhar, J. A. Rogers, and K. A. Nelson, Time-resolved vibrational spectroscopy in the impulsive limit, Chem. Rev. 94, 157 (1994); R. Boyd, Nonlinear Optics (Academic, New York, 2008); S. Mukamel, Principles of Nonlinear Optics and Spectroscopy (Oxford University Press, New York, 1995).

[10] S. T. Cundiff and S. Mukamel, Optical multidimensional coherent spectroscopy, Phys. Today 66, No. 7, 44 (2013).

[11] F. Bencivenga et al., Four-wave mixing experiments with extreme ultraviolet transient gratings, Nature (London) 520, 205 (2015).

[12] A. M. Kondratenko and E. L. Saldin, Generation of coherent radiation by a relativistic electron beam in an undulator, Part. Accel. 10, 207 (1980), https://inspirehep.net/files/ 872da099a0e9c171a4dca19256f7ca0e.

[13] R. Bonifacio, C. Pellegrini, and L. Narducci, Collective instabilities and high-gain regime in a free electron laser, Opt. Commun. 50, 373 (1984).

[14] G. Geloni, V. Kocharyan, and E. Saldin, Generation of doublet spectral lines at self-seeded x-ray FELs, Opt. Commun. 284, 3348 (2011).

[15] A. A. Lutman, F.-J. Decker, J. Arthur, M. Chollet, Y. Feng, J. Hastings, Z. Huang, H. Lemke, H.-D. Nuhn, A. Marinelli, J. L. Turner, S. Wakatsuki, J. Welch, and D. Zhu, Demonstration of Single-Crystal Self-Seeded TwoColor X-ray Free-Electron Lasers, Phys. Rev. Lett. 113, 254801 (2014).

[16] D. Ratner et al., Experimental Demonstration of a Soft X-Ray Self-Seeded Free-Electron Laser, Phys. Rev. Lett. 114, 054801 (2015).

[17] E. Allaria et al., Highly coherent and stable pulses from the FERMI seeded free-electron laser in the extreme ultraviolet, Nat. Photonics 6, 699 (2012).

[18] E. Allaria et al., Two-stage seeded soft-x-ray free-electron laser, Nat. Photonics 7, 913 (2013).

[19] B. Liu et al., Demonstration of a widely-tunable and fullycoherent high-gain harmonic-generation free-electron laser, Phys. Rev. Accel. Beams 16, 020704 (2013).

[20] L.-H. Yu, Generation of intense UV radiation by subharmonically seeded single-pass free-electron lasers, Phys. Rev. A 44, 5178 (1991).

[21] G. Stupakov, Using the Beam-Echo Effect for Generation of Short-Wavelength Radiation, Phys. Rev. Lett. 102, 074801 (2009).

[22] P. R. Ribič et al., Coherent soft x-ray pulses from an echoenabled harmonic generation free-electron laser, Nat. Photonics 13, 555 (2019).

[23] L.-H. Yu and T. Shaftan, Towards coherent X-ray freeelectron lasers, Nat. Photonics 13, 513 (2019).

[24] E. L. Saldin, E. A. Schneidmiller, and M. V. Yurkov, Klystron instability of a relativistic electron beam in a bunch compressor, Nucl. Instrum. Methods Phys. Res., Sect. A 490, 1 (2002).

[25] S. Di Mitri, Coherent synchrotron radiation, and microbunching instability, in Proceedings of the CERN Accelerator School: Free Electron Lasers and Energy Recovery Linacs, Hamburg, Germany (2016), edited by R. Bailey, CERN Yellow Reports: School Proceedings, Vol. 1/2018, CERN-2018-001-SP (CERN, Geneva, 2018).

[26] J. Wu, P. Emma, Z. Huang, and C. Limborg, Temporal profile of the LCLS photocathode ultraviolet drive laser 
tolerated by the microbunching instability, Reports No. SLAC-PUB-10430 and No. LCLS-TN-04-6, 2004.

[27] R. Bonifacio, B. W. McNeil, and P. Pierini, Superradiance in the high-gain free-electron laser, Phys. Rev. A 40, 4467 (1989).

[28] G. Marcus, W. M. Fawley, D. Bohler, Y. Ding, Y. Feng, Z. Huang, J. Krzywinski, A. Lutman, and D. Ratner, Experimental observations of seed growth and accompanying pedestal contamination in a self-seeded, soft x-ray freeelectron laser, Phys. Rev. Accel. Beams 22, 080702 (2019).

[29] E. Hemsing, A. Havalanau, and Z. Zhang, Statistical theory of a self-seeded free electron laser with noise pedestal growth, Phys. Rev. Accel. Beams 23, 010701 (2020).

[30] Z. Zhang, G. Marcus, E. Hemsing, W. M. Fawley, Z. Huang, and A. Lutman, Statistical analysis of a self-seeded $\mathrm{X}$-ray free-electron laser in the presence of the microbunching instability, Phys. Rev. Accel. Beams 23, 010704 (2020).

[31] E. Roussel, E. Ferrari, E. Allaria, G. Penco, S. Di Mitri, M. Veronese, M. Danailov, D. Gauthier, and L. Giannessi, Multicolor High-Gain Free-Electron Laser Driven by Seeded Microbunching Instability, Phys. Rev. Lett. 115, 214801 (2015).

[32] C. Behrens, Z. Huang, and D. Xiang, Reversible electron beam heating for suppression of microbunching instabilities at free-electron lasers, Phys. Rev. Accel. Beams 15, 022802 (2012).

[33] J. Qiang, C. E. Mitchell, and M. Venturini, Suppression of Microbunching Instability Using Bending Magnets in Free-Electron-Laser Linacs, Phys. Rev. Lett. 111, 054801 (2013).

[34] S. Di Mitri and S. Spampinati, Microbunching Instability Suppression via Electron-Magnetic-Phase Mixing, Phys. Rev. Lett. 112, 134802 (2014).

[35] C. Feng, D. Huang, H. Deng, Q. Gu, and Z. Zhao, Suppression of microbunching instability via a transverse gradient undulator, New J. Phys. 17, 073028 (2015).

[36] Z. Huang et al., Measurements of the linac coherent light source laser heater and its impact on the x-ray free-electron laser performance, Phys. Rev. Accel. Beams 13, 020703 (2010).

[37] S. Spampinati et al., Laser heater commissioning at an externally seeded free-electron laser, Phys. Rev. Accel. Beams 17, 120705 (2014).

[38] J. Lee, J.-H. Han, S. Lee, J. Hong, C. H. Kim, C. K. Min, and I. S. Ko, PAL-XFEL laser heater commissioning, Nucl. Instrum. Methods Phys. Res., Sect. A 843, 39 (2017).

[39] E. L. Saldin, E. A. Schneidmiller, and M. Yurkov, Longitudinal space charge-driven microbunching instability in the TESLA Test Facility linac, Nucl. Instrum. Methods Phys. Res., Sect. A 528, 355 (2004).

[40] E. Ferrari, E. Allaria, W. Fawley, L. Giannessi, Z. Huang, G. Penco, and S. Spampinati, Impact of Non-Gaussian Electron Energy Heating upon the Performance of a Seeded Free-Electron Laser, Phys. Rev. Lett. 112, 114802 (2014).

[41] J. Tang et al., Laguerre-Gaussian Mode Laser Heater for Microbunching Instability Suppression in Free-Electron Lasers, Phys. Rev. Lett. 124, 134801 (2020).

[42] C.-Y. Tsai, S. Di Mitri, D. Douglas, R. Li, and C. Tennant, Conditions for coherent-synchrotron-radiation-induced microbunching suppression in multibend beam transport or recirculation arcs, Phys. Rev. Accel. Beams 20, 024401 (2017).

[43] Ya. S. Derbenev, J. Rossbach, E. L. Saldin, and V. D. Shiltsev, Microbunch radiative tail-head interaction, Report No. TESLA-FEL 95-05, DESY, Hamburg, Germany, 1995.

[44] S. Heifets, G. Stupakov, and S. Krinsky, Coherent synchrotron radiation instability in a bunch compressor, Phys. Rev. Accel. Beams 5, 064401 (2002).

[45] S. Di Mitri, M. Cornacchia, and S. Spampinati, Cancellation of Coherent Synchrotron Radiation Kicks with Optics Balance, Phys. Rev. Lett. 110, 014801 (2013).

[46] R. A. Bosch, K. J. Kleman, and J. Wu, Modeling two-stage bunch compression with wakefields: Macroscopic properties and microbunching instability, Phys. Rev. Accel. Beams 11, 090702 (2008).

[47] S. Di Mitri et al., Experimental evidence of intrabeam scattering in a free-electron laser driver, New J. Phys 22, 083053 (2020).

[48] C.-Y. Tsai, Concatenated analyses of space microbunching in high brightness electron beam transport, Nucl. Instrum. Methods Phys. Res., Sect. A 940, 462 (2019).

[49] D. Ratner, C. Behrens, Y. Ding, Z. Huang, A. Marinelli, T. Maxwell, and F. Zhou, Time-resolved imaging of the microbunching instability and energy spread at the Linac Coherent Light Source, Phys. Rev. Accel. Beams 18, 030704 (2015).

[50] M. Venturini and J. Qiang, Transverse space-charge induced microbunching instability in high-brightness electron bunches, Phys. Rev. Accel. Beams 18, 054401 (2015).

[51] K. Zhang, L. Zeng, Z. Qi, C. Feng, and D. Wang, Eliminating the microbunching-instability-induced sideband instability in a soft x-ray self-seeding free-electron laser, Nucl. Instrum. Methods Phys. Res., Sect. A 882, 22 (2018). 